\title{
US increases Ebola screening at five airports
}

\author{
Michael McCarthy \\ Seattle
}

The United States will increase its screening of passengers for signs of Ebola infection at five international airports, officials from the Centers for Disease Control and Prevention (CDC) and the Department of Homeland Security's Customs and Border Protection announced on Wednesday 8 October.

The screening measures will begin by the end of this week at New York's John F Kennedy Airport and then at Washington Dulles, Newark, Chicago O'Hare, and Atlanta airports the following week, said Tom Frieden, CDC director. "We believe these new measures will further protect the health of Americans, understanding that nothing we can do will get us to absolute zero risk until we end the Ebola epidemic in west Africa," he said.

Nearly half of travelers from the three nations most affected by the current Ebola outbreak in west Africa-Guinea, Liberia, and Sierra Leone-enter through the New York airport, and altogether more than $94 \%$ of travelers from these countries enter the US through the five airports selected for enhanced screening, Frieden said.

Currently, all outbound passengers are screened in these three countries before they are allowed to board aircraft. Over the past two months these programs have screened 36000 passengers, 77 of whom were denied boarding because of fever or their response to questions. Many had malaria, Frieden said, but none had Ebola.

Under the new protocol all travelers from Guinea, Liberia, or Sierra Leone will be escorted to an area of the airport set up for screening. There they will be asked a series of questions about their health and possible Ebola exposure, have their temperatures taken with a non-contact thermometer, and be observed for symptoms. Only about 150 travelers from the affected countries arrive in the US each day.

Travelers with fever or symptoms or whose response to the health questionnaire suggests possible Ebola exposure will then be evaluated by a CDC quarantine station public health officer. Suspected cases will be handed to local healthcare systems where they will be further assessed and, if necessary, isolated. Other people who may have been in contact with someone with Ebola will be referred to the local public health authorities, which will monitor their temperatures daily for 21 days after their last exposure (the incubation period for Ebola infection). All passengers arriving from those three countries will also be required to supply information about how they can be contacted by the CDC while they are in the US. Currently, as standard procedure, Customs and Border Protection and CDC officers observe all passengers arriving in the US for signs of illness and question any who appear ill.

Frieden said that a ban on flights from west Africa - which several politicians have called for-would make the situation worse in Africa and, ultimately, in the US. He said, "The problem with that approach is that it makes it extremely difficult to respond to the outbreak. It makes it hard to get health workers in, because they can't get out. If we make it harder to respond to the outbreak in west Africa, it will spread not only in those three countries but to other parts of Africa and will ultimately increase the risk here."

To date, more than 3700 people have died from Ebola since the outbreak was first reported in March of this year.

Cite this as: BMJ 2014;349:g6147

๑ B BMJ Publishing Group Ltd 2014 